\title{
A SUFFICIENT CONDITION FOR CESÀRO SUMMABILITY
}

\author{
G. D. NICHOLS
}

S. Chapman ${ }^{1}$ has proved the summability $(C, k)$ of the series $\sum r^{s} e^{r i \theta}$ where $(k-1) \leqq s<k$, and $0<\theta<2 \pi$. More recently M. S. MacPhail ${ }^{2}$ has proved the exact summability $(C, k)$ of the series $\sum P(r) \cdot f(r)$, where $P(r)$ is a polynomial of degree $k-1$ and $f(r)$ is a periodic function of mean value zero. He also gave a closed expression for the Cesàro "sum" of such a series. H. L. Garabedian ${ }^{3}$ earlier published a special case of this result.

The purpose of the present paper is to prove the following closely related theorem in which the condition on the coefficients is somewhat more general. Also "sum" formulas are given for each of the sine and cosine series separately.

THEOREM. The series $(1 / 2) P(0)+\sum P(r) \cos r x$ and $\sum P(r) \sin r x$ are summable $(C, k)$ provided $\left\{\Delta^{k} P(r)\right\}$ is a monotone null sequence, and $P(r)$ together with its first $k+1$ derivatives each exist and are continuous for positive values of $r(x \neq 2 n \pi$ in the cosine series).

The proofs for the sine and cosine series are almost identical so only the proof for the latter will be given. Consider the series

$$
S_{n}=(1 / 2) P(0)+\sum_{r=1}^{n} P(r) \cos r x .
$$

If we multiply both sides of (1) successively $k$ times by $2 \sin x / 2$ there results, for $k$ even,

$$
\begin{aligned}
\left(2^{k} \sin ^{k} x / 2\right) S_{n}= & (1 / 2) D_{0 k}+\sum_{r=1}^{(k-2) / 2} D_{r k} \cos r x \\
& +(-1)^{k / 2} \sum_{r=0}^{n-k} \Delta^{k} P(r) \cos (r+(k / 2)) x+(\operatorname{Cos})_{k},
\end{aligned}
$$

where the symbol $(\mathrm{Cos})_{k}$ represents $k$ cosine terms, the highest order of any of the coefficients being the same as the order of $P(n)$.

For $k$ odd, we have 1941.

Presented to the Society, January 1, 1941; received by the editors October 20,

${ }^{1}$ S. Chapman, Proceedings of the London Mathematical Society, (2), vol. 9 (1911), p. 398.

2 M. S. MacPhail, this Bulletin, vol. 47 (1941), p. 483.

${ }^{3}$ H. L. Garabedian, this Bulletin, vol. 45 (1939), p. 592. 


$$
\begin{aligned}
\left(2^{k} \sin ^{k} x / 2\right) S_{n}= & \sum_{r=1}^{(k-2) / 2} C_{r k} \sin (2 r-1) x / 2 \\
& +(-1)^{(k+1) / 2} \sum_{r=0}^{n-k} \Delta^{k} P(r) \sin (r+(k / 2)) x+(\operatorname{Sin})_{k},
\end{aligned}
$$

where $(\operatorname{Sin})_{k}$ has the same significance as $(\operatorname{Cos})_{k}$ above. For $k=1$ the first summation in (3) is to be omitted. The expressions for $D_{r k}$ and $C_{r k}$ in (2) and (3), respectively, are given in the sequel.

If we now apply the Cesàro definition for the "sum," $\sigma$, of a nonconvergent series, there results from (2),

$$
\begin{aligned}
& \left(2^{k} \sin ^{k} x / 2\right) \sigma=(1 / 2) D_{0 k}+\sum_{r=1}^{(k-2) / 2} D_{r k} \cos r x \\
& +\lim _{n \rightarrow \infty}(-1)^{k / 2} \sum_{r=0}^{n-k} \frac{C(n-r+(k / 2)+1, k)}{C(n+k, k)} \Delta^{k} P(r) \cos (r+(k / 2)) x \\
& +\lim _{n \rightarrow \infty} \frac{(\operatorname{Cos})_{k}}{(n+1)(n+2) \cdots(n+k)},
\end{aligned}
$$

where the $C$ 's following the second summation are the binomial coefficients.

To prove the existence of the first limit in (4) we first note that since there are the same number of factors in numerator and denominator of the fraction preceding $\Delta^{k} P(r)$, the fraction is positive and never greater than one for the values of $n, r$, and $k$ under consideration. Since $\left\{\Delta^{k} P(r)\right\}$ is a monotone null sequence by hypothesis, the existence of the first limit (except for $x=2 n \pi$ ) follows by Dirichlet's test. ${ }^{4}$

We wish to show that the second limit in (4) is equal to zero. Since the order of $(\operatorname{Cos})_{k}$ is the same as $P(n)$, this can be done by showing that if $\left\{\Delta^{k} P(n)\right\}$ is a monotone null sequence then the $k$ th derivative of $P(n)$ approaches zero as $n$ becomes infinite.

For this purpose we have

$$
P^{(k)}(n)+(k / 2) P^{(k+1)}(\xi)=\Delta^{k} P(n),
$$

where $\xi \rightarrow \infty$ as $n \rightarrow \infty$. This is a special case of a formula due to Markoff, ${ }^{5}$ and depends on the continuity of $P(n)$ and its first $k+1$ derivatives.

Since, by hypothesis, $\Delta^{k} P(n) \rightarrow 0$ as $n \rightarrow \infty$, it is only necessary that if $\left(y+(k / 2) y^{\prime}\right) \rightarrow 0$ as $n \rightarrow \infty, y$ and $y^{\prime}$ separately do so, where $y=P^{(k)}(n)$, and $y$ and $y^{\prime}$ are continuous. A proof of this fact is given by Brom-

${ }^{4}$ Bromwich, Theory of Infinite Series, p. 49.

${ }^{5}$ Markoff, Differenzenrechnung, p. 21. 
wich. ${ }^{6}$ Hence the summability of the original series is established.

In case $P(r)$ is a polynomial of degree $k-1$, the first limit in (4) is also zero and $\sigma$ is obtained in closed form from the first summation. Formulas for $D_{r k}$ and $C_{r k}$ are now given for this case. They can be established by induction, but the details are rather tedious and will be omitted

$$
\begin{aligned}
D_{r k}=\sum_{x=k / 2+r-1}^{k-2}(-1)^{x+k+r+1} \Delta^{k-1-x} P(0) & {[C(x,(k / 2)+r-1)} \\
& +C(x,(k / 2)-r-1)] .
\end{aligned}
$$

For $k$ odd, and $P(r)$ a polynomial of degree $k-1$, there results from (3),

(7) $\left(2^{k} \sin ^{k} x / 2\right) \sigma=\sum_{r=1}^{(k-1) / 2} C_{r k} \sin (2 r-1) x / 2$,

$$
\begin{aligned}
C_{r k}=\sum_{x=(k-2 r-1) / 2}^{k-2}(-1)^{x+k+r} \Delta^{k-1-x} P(0)[C(x,(k / 2) \\
+r-(3 / 2))+C(x,(k / 2)-r-(1 / 2))] .
\end{aligned}
$$

For the sine series, $\sum P(r) \sin r x$, the results for $P(r)$, a polynomial of degree $k-1$, follow. For $k$ odd,

$$
\left(2^{k} \sin ^{k} x / 2\right) \sigma=\sum_{r=1}^{(k-1) / 2} A_{r k} \cos (2 r-1) x / 2,
$$

and for $k$ even,

$$
\left(2^{k} \sin ^{k} x / 2\right) \sigma=\sum_{r=1}^{(k-2) / 2} B_{r k} \sin r x,
$$

where $A_{r k}$ and $B_{r k}$ are given by

$$
\begin{array}{r}
A_{r k}=\sum_{x=(k-2 r-1) / 2}^{k-3}(-1)^{x+k+r-1} \Delta^{k-1-x} P(0)[C(x,(k / 2)-r-(1 / 2)) \\
-C(x,(k / 2)+r-(3 / 2))] \\
B_{r k}=\sum_{x=(k-2 r-2) / 2}^{k-3}(-1)^{x+k+r-1} \Delta^{k-1-x} P(0)[C(x,(k / 2)-r-1) \\
-C(x,(k / 2)+r-1)] .
\end{array}
$$

If the upper limit on $\sum$ in (4), (7), (9), and (10) is less than the lower limit, the value of the summation is to be taken as zero. Also $C(n, 0)=1$ for all $n$ including zero, and $C(n, m)=0$ for $n<m$.

\section{University of ARKansas}

${ }^{6}$ Bromwich, Theory of Infinite Series, p. 272. 